\title{
GENERICITY OF SIMPLE EIGENVALUES FOR ELLIPTIC PDE'S
}

\section{J. H. ALBERT}

ABSTRACT. The spectrum of a selfadjoint, $C^{\infty}$ linear elliptic par tial differential operator on a compact manifold contains only isolated eigenvalues, each having finite multiplicity. It is sometimes the case that these multiplicities are unbounded; this is common in problems arising in applications because of the high degree of symmetry usually present. The main theorem shows that the property of having only simple eigenvalues is generic for operators obtained by varying the zeroth order part of a given operator.

The purpose of this article is to provide a proof of the following theorem.

Main theorem. Let $M$ be a compact, connected $C^{\infty}$ manifold without boundary. Let $L$ be a selfadjoint, $C^{\infty}$ linear elliptic differential operator on $M$. The set $\left\{\rho \in C^{\infty}(M)\right.$ : all eigenvalues of $L+\rho$ are simple $\}$ is residual in $C^{\infty}(M)$.

The term elliptic operator used in this article will always mean the operator is selfadjoint, $C^{\infty}$ and linear. The main theorem can be summarized by saying that almost all elliptic operators obtained by varying the zeroth order part of a given operator have only simple eigenvalues. For terminology, see $\$ 1$.

The main theorem was first announced in [1] for second-order operators, and the proof is the one given in [2]; it uses perturbation theory. A proof using transversality theory, applicable to operators whose eigenfunctions satisfy the strong unique continuation property, has been obtained by $\mathrm{K}$. Uhlenbeck [6]. The theorem is particularly useful in obtaining genericity results about the eigenfunctions (see [1], [2], [6]).

1. Notation; outline of proof. The manifold $M$ and the operator $L$ will be fixed throughout as in the theorem. For standard terminology on elliptic operators, see [3], [4].

Received by the editors June 4, 1973 and, in revised form, January 23, 1974. AMS (MOS) subject classifications (1970). Primary 35P05, 35J30; Secondary 47A55, 47B25, 58G99.

Key words and phrases. Elliptic operator, eigenvalue, generic, perturbation. 
Eigenvalue properties. Given an elliptic operator $P$, and $\lambda \in \mathbf{R}$, let $h(\lambda)=\operatorname{dim} \operatorname{ker}(P-\lambda) . \lambda$ is an eigenvalue of $P$ if $h(\lambda)>0 ; \lambda$ is simple if $h(\lambda)=1 . h(\lambda)$ is a positive integer, the multiplicity of the eigenvalue $\lambda$. The set of eigenvalues of $P$ is countable, has no finite accumulation points, and is bounded from below (with appropriate normalization of $P$ ). Thus the eigenvalues can be written as an increasing sequence $\lambda_{1} \leq \lambda_{2} \leq$ $\cdots \leq \lambda_{n} \leq \cdots \rightarrow+\infty$, in which an eigenvalue of multiplicity $h$ is repeated $h$ times. It makes sense to talk about the first $n$ eigenvalues of $P$.

Topological notions. If $X$ is a topological space, $A \subset X$ is residual in $X$ if $A$ is a countable intersection of open, dense sets in $X$. If $A$ is residual, then $A$ is dense in $X$; in particular, $A$ is nonempty if $X$ is. $A$ may or may not be open. The complement of $A$ is a set of first category in $X$.

Here $X=C^{\infty}(M)$. The topology is that induced on $C^{\infty}(M)$ by the countable family of $C^{k}$ norms on $M$. If $U \subset \mathbf{R}^{\nu}, u \in C_{0}^{\infty}(U)$, then

$$
|u|_{k}=\sup _{x \in U} \sum_{|a|_{\leq k}}\left|D^{a} u(x)\right|
$$

gives the $C^{k}$ norm on $U$, and this is globalized to the norm $|\cdots|_{k}$ on $M$ by a partition of unity.

Outline of proof. 'Let $A=\left\{\rho \in C^{\infty}(M)\right.$ : all eigenvalues of $L+\rho$ are simple $\}$ and $A_{n}=\left\{\rho \in C^{\infty}(M)\right.$ : the first $n$ eigenvalues of $L+\rho$ are simple $\}$; $A_{0}=C^{\infty}(M)$. Then

$$
A \subset \cdots \subset A_{n} \subset A_{n-1} \subset \cdots \subset A_{2} \subset A_{1} \subset A_{0}^{\prime}=C^{\infty}(M)
$$

and $A=\bigcap_{n=0}^{\infty} A_{n}$.

Theorem 1. $A_{n}$ is open in $C^{\infty}(M)$, for all $n=0,1,2, \ldots$

Theorem 2. $A_{n+1}$ is dense in $A_{n}$, for all $n=0,1,2, \ldots$.

Theorems 1 and 2 imply $A_{n}$ is open and dense in $C^{\infty}(M)$, hence $A$ is residual. Theorem 1 is essentially the stability of simple eigenvalues under small perturbations of the zeroth order term of the operator. This is not a new result (see [4]) but the proof is given $(\$ 2)$ for completeness. Theorem 2 depends on a perturbation theorem due to Rellich [5]. It is proved in $\$ 3$.

\section{Stability of simple eigenvalues.}

Minimax principle. Given an elliptic operator $P$ on $M$, define a quadratic form on $C^{\infty}(M)$ by $\Lambda(u)=\int u(P u)$. Note. All integrals are taken with respect to a fixed smooth measure on $M$. Given a linearly independent set $\left\{v_{1}, \cdots, v_{n-1}\right\}$ of $C^{\infty}$ functions on $M$, let 


$$
\begin{aligned}
& \Lambda_{n}\left(v_{1}, \ldots, v_{n-1}\right)=\min \left\{\Lambda(u): u \in C^{\infty}(M), \int u^{2}=1,\right. \\
& \left.\qquad u v_{i}=0 \text { for } 1 \leq i \leq n-1\right\} .
\end{aligned}
$$

Then: $\lambda_{n}=\max \left\{\Lambda_{n}\left(v_{1}, \cdots, v_{n-1}\right):\left\{v_{1}, \cdots, v_{n-1}\right\} \subset C^{\infty}(M)\right.$ is linearly independent $\}$.

Proposition (Continuity of the eigenvalues). Let $\rho, \rho^{\prime} \in C^{\infty}(M)$ and let $\lambda_{n}, \lambda_{n}^{\prime}$ be the nth eigenvalues of $L+\rho$ and $L+\rho^{\prime}$ respectively. Then $\left|\lambda_{n}^{\prime}-\lambda_{n}\right| \leq\left|\rho^{\prime}-\rho\right|_{0}$.

Proof. Let $\Lambda(u)=\int u[(L+\rho) u]$ and $\Lambda^{\prime}(u)=\int u\left[\left(L+\rho^{\prime}\right) u\right]$ be the quadratic forms of the minimax principle corresponding to $\rho$ and $\rho^{\prime}$.

$$
\left|\Lambda^{\prime}(u)-\Lambda(u)\right|=\left|\int\left(\rho^{\prime}-\rho\right) u^{2}\right| \leq\left|\rho^{\prime}-\rho\right|_{0} \int u^{2}=\left|\rho^{\prime}-\rho\right|_{0} \quad \text { if } \int u^{2}=1,
$$

i.e.

$$
\Lambda(u)-\left|\rho^{\prime}-\rho\right|_{0} \leq \Lambda^{\prime}(u) \leq \Lambda(u)+\left|\rho^{\prime}-\rho_{0}\right| \text { for } \int u^{2}=1 .
$$

Taking the min over $u \perp v_{1}, \cdots, v_{n-1}$, and then the max over all allowable $\left\{v_{1}, \cdots, v_{n-1}\right\}$, and applying the minimax principle y.ields

$$
\lambda_{n}-\left|\rho^{\prime}-\rho\right|_{0} \leq \lambda_{n}^{\prime} \leq \lambda_{n}+\left|\rho^{\prime}-\rho\right|_{0},
$$

i.e. $\left|\lambda_{n}^{\prime}-\lambda_{n}\right| \leq\left|\rho^{\prime}-\rho\right|_{0}$ as required.

Proof of Theorem 1. $A_{n}$ is open in $C^{\infty}(M)$.

Let $\rho \in A_{n}$ be given, and let $\lambda_{1}<\lambda_{2}<\cdots<\lambda_{n}<\lambda_{n+1} \leq \cdots$ be the eigenvalues of $L+\rho$; the first $n$ are simple. Let $\delta=\min \left\{\lambda_{j+1}-\lambda_{j}: j=\right.$ $1, \cdots, n\} ; \delta>0$. Let $U=\left\{\rho^{\prime} \in C^{\infty}(M):\left|\rho^{\prime}-\rho\right|_{0}<\delta / 2\right\} ; U$ is an open nbd of $\rho$. Let $\left\{\lambda_{j}^{\prime}\right\}$ be the eigenvalues of $L+\rho^{\prime}$ for $\rho^{\prime} \in U$. Then, by the Proposition above,

$$
\left|\lambda_{j}^{\prime}-\lambda_{j}\right| \leq\left|\rho^{\prime}-\rho\right|_{0}<\delta / 2 \quad \text { for all } j .
$$

Claim $\lambda_{j}^{\prime} \neq \lambda_{j+1}^{\prime}$ for $j=1, \cdots, n$. We have

$$
\begin{aligned}
\delta & \leq \lambda_{j+1}-\lambda_{j} \leq\left|\lambda_{j+1}^{\prime}-\lambda_{j+1}\right|+\left|\lambda_{j}^{\prime}-\lambda_{j+1}^{\prime}\right|+\left|\lambda_{j}^{\prime}-\lambda_{j}\right| \\
& <\delta / 2+\left|\lambda_{j}^{\prime}-\lambda_{j+1}^{\prime}\right|+\delta / 2=\delta+\left(\lambda_{j+1}^{\prime}-\lambda_{j}^{\prime}\right),
\end{aligned}
$$

implying $\lambda_{j+1}^{\prime}-\lambda_{j}^{\prime}>0, j=1, \cdots, n$. Hence the first $n$ eigenvalues of $L+\rho^{\prime}$ are simple if $\rho^{\prime} \in U$, so $U \subset A_{n}$, imply.ing $A_{n}$ is open. 
3. Density arguments. Let $f(\tau)$ be a function defined on a nbd of $\tau=0$ in $\mathbf{R}$, with values in $C^{\infty}(M)$. $f$ is analytic in $C^{\infty}(M)$ at $\tau=0$ if there is a sequence $\left\{f_{i}\right\}$ of elements of $C^{\infty}(M)$ such that $\lim _{m \rightarrow \infty}\left|f(\tau)-\sum_{i=0}^{m} f_{i} \tau^{i}\right|_{k}=0$ for $\tau$ in a nbd of $0, k=0,1,2, \ldots f(\tau)$ is regular at $\tau=0$ if, in addition, the real series $\sum_{i=0}^{\infty}\left|f_{i}\right|_{k}|\tau|^{i}$ converges for $\tau$ in a nbd of $0, k=0,1,2, \cdots$

Perturbation theorem. Let $\lambda$ be an eigenvalue of multiplicity $h$ of $L+\rho$. Let $\rho(\tau)$ be regular in $C^{\infty}(M)$ at $\tau=0$, with $\rho(0)=\rho$. Then there are $b$ functions $\lambda^{(1)}(\lambda), \cdots, \lambda^{(b)}(\lambda)$, real-analytic at $\tau=0$, and $h$ functions $u^{(1)}(\tau), \cdots, u^{(h)}(r)$, analytic in $C^{\infty}(M)$ at $\tau=0$ such that:

(a) $\lambda^{(i)}(0)=\lambda, i=1, \cdots, h$;

(b) for $\tau$ in a small enough nbd of $0,\left\{u^{(1)}(\tau), \ldots, u^{(b)}(\tau)\right\}$ is an orthonormal set in $L_{2}(M)$ and $u^{(i)}(r)$ is an eigenfunction of $L+\rho(r)$ with eigenvalue $\lambda^{(i)}(r)$;

(c) for every open interval $(a, b) \subset \mathbf{R}$ such that $\lambda$ is the only eigenvalue of $L+\rho$ in $[a, b]$, there are exactly $b$ eigenvalues (counting multiplicity) $\lambda^{(1)}(\tau), \ldots, \lambda^{(b)}(\tau)$ of $L+\rho(\tau)$ in $(a, b)$, for $\tau$ small enough.

This theorem is a minor variation of a theorem due to F. Rellich [5]. The proof there is a Hilbert space argument and only gives analyticity of the eigenfunctions in $L_{2}(M)$. However, the basic equation in the argument can be iterated to give analyticity in the space $H^{s}(M)$ of functions with $L_{2}$-derivatives up to order $s$, and Sobolev's lemma then gives analyticity in $C^{\infty}(M)$. ([2, Appendix II] contains more details.)

One can write down explicit expressions for the coefficients of these power series by equating coefficients in the equations

$$
\left(L+\rho(\tau)-\lambda^{(j)}(\tau)\right) u^{(j)}(\tau)=0 .
$$

However, rather than using the entire power series, we will only need the first two terms. We will write $\lambda^{(j)}(\tau)=\lambda+\tau \alpha_{j}+\tau^{2} \beta_{j}(\tau)$ for the perturbed eigenvalues, where $a_{j} \in \mathbf{R}$ and $\beta_{j}(\tau)$ is real-analytic at $\tau=0, u^{(j)}(\tau)=$ $u_{j}+\pi v_{j}+\tau^{2} w_{j}(\tau)$ for the perturbed eigenfunctions, where $u_{j}, v_{j} \in C^{\infty}(M)$ and $w_{j}(\tau)$ is analytic in $C^{\infty}(M)$ at $\tau=0$. Also we will only need linear perturbations $\rho(\tau)=\rho+\tau \sigma$, where $\sigma \in C^{\infty}(M)$.

Differentiating the equation (1) with respect to $\tau$ and setting $\tau=0$ y.ields

$$
(L+\rho-\lambda) v_{j}+\left(\sigma-\alpha_{j}\right) u_{j}=0, \quad j=1, \cdots, h .
$$

From this it follows that $\left(\sigma-\alpha_{j}\right) u_{j} \perp \operatorname{ker}(L+\rho-\lambda), j=1, \cdots, h$, implying 
$\int\left(\sigma-\alpha_{j}\right) u_{j} u_{k}=0, j, k=1, \cdots, h, \quad$ and

$$
\int \sigma u_{j} u_{k}=\alpha_{j} \delta_{j k}
$$

where $\delta_{j k}$ is the Kronecker delta.

Proof of Theorem 2. $A_{n+1}$ is dense in $A_{n}, n=0,1,2, \ldots$.

Assume that $\rho \in A_{n}$ is given; it is necessary to produce $\rho^{\prime} \in A_{n+1}$ arbitrarily close to $\rho$. Suppose that $\lambda_{1}<\lambda_{2}<\cdots<\lambda_{n}<\lambda_{n+1}=\cdots=$ $\lambda_{n+h}<\lambda_{n+b+1} \leq \cdots$ are the eigenvalues of $L+\rho$. The first $n$ are simple, and will remain simple under small perturbations of $\rho$, by Theorem 1 . The $(n+1)$ st eigenvalue $\lambda\left(=\lambda_{n+1}=\cdots=\lambda_{n+b}\right)$ has multiplicity $h$. We will show that there is a linear perturbation $\rho(\tau)=\rho+\tau \sigma$ of $\rho$ such that the $(n+1)$ st eigenvalue of $L+\rho(\tau)$ has multiplicity at most $h-1$ if $\tau$ is small enough and $\neq 0$. By a finite sequence of perturbations of this type, the $(n+1)$ st eigenvalue can be made simple.

Given $\sigma \in C^{\infty}(M), \rho(\tau)=\rho+\tau \sigma$ induces perturbed eigenvalues $\lambda^{(j)}(\tau)=$ $\lambda+\tau \alpha_{j}+\tau^{2} \beta_{j}(\tau)$ and eigenfunctions $u^{(j)}(\tau)=u_{j}+\tau v_{j}+\tau^{2} w_{j}(\tau)$ for $j=1, \ldots$, $h$. Observe that if $\alpha_{j} \neq \alpha_{k}$ for some $j, k$, then $\lambda^{(j)}(\tau) \neq \lambda^{(k)}(r)$ for $\tau$ small enough and $\neq 0$. Since the multiplicity of the $(n+1)$ st eigenvalue of $L+$ $\rho(r)$ is $\leq h$ by (c) of the perturbation theorem, the fact that two of the $h$ perturbed eigenvalues are distinct implies it is $\leq h-1$ and we are done. The possibility of making two $a_{j}$ 's distinct is a consequence of the equations (3), and is proved in the

Lemma. There exists $\sigma \in C^{\infty}(M)$ such that for the perturbation $\rho(\tau)=$ $\rho+\tau \sigma$, at least two of the $\alpha_{j}$ are distinct.

Proof. Let $N=\operatorname{ker}(L+\rho+\lambda) . N$ is a vector space of dimension $h$ contained in $C^{\infty}(M)$, so $N$ has an inner product $\langle f, g\rangle=\int f g$. Given $\sigma \epsilon$ $C^{\infty}(M)$, there is a quadratic form on $N$ given by $(f, g) \rightarrow \int \sigma f g$, so there exists a selfadjoint linear operator $G_{\sigma}: N \rightarrow N$ such that $\left\langle G_{\sigma} f, g\right\rangle=\int \sigma f g$, for all f. $g \in N$. Furthermore, given any orthonormal basis $\left\{f_{1}, \ldots, f_{h}\right\}$ of $N$, the matrix of $G_{\sigma}$ with respect to this basis has $\left\langle G_{\sigma} f_{j}, f_{k}\right\rangle$ as its $j k$ th entry. Once $\sigma$ is given, we have the orthonormal basis $\left\{u_{1}, \ldots, u_{h}\right\}$ of unperturbed eigenfunctions. In this basis, $\left\langle G_{\sigma} u_{j}, u_{k}\right\rangle=\int \sigma u_{j} u_{k}=\alpha_{j} \delta_{j k}$ by (3), so the matrix of $G_{\sigma}$ is diagonal and its diagonal entries are $\alpha_{1}, \ldots, \alpha_{b}$. If $\alpha_{1}=$ $\cdots=\alpha_{b}(=\alpha)$, then $G_{\sigma}=\alpha I$ in this basis and hence in any basis. To choose $\sigma$ so this does not happen, fix a basis $\left\{f_{1}, \cdots, f_{b}\right\}$ of $N$. If $f_{1} f_{2} \not \equiv$ 0 , let $\sigma=f_{1} f_{2}$. Then the matrix of $G_{\sigma}$ has an entry $\int \sigma f_{1} f_{2}=\int\left(f_{1} f_{2}\right)^{2} \neq 0$ off the diagonal and thus cannot be a multiple of the identity. Similarly if 
$f_{1} f_{2} \equiv 0$, which is possible for operators not satisfying the unique continuation property, take $\sigma=f_{1}^{2}-f_{2}^{2}$. Then the corresponding entry of $G_{\sigma}$ in the basis $\left\{f_{1}+f_{2}, f_{1}-f_{2}, f_{3}, \cdots, f_{n}\right\}$ is

$$
\int \sigma\left(f_{1}+f_{2}\right)\left(f_{1}-f_{2}\right)=\int\left(f_{1}^{4}+f_{2}^{4}\right)
$$

since $f_{1} f_{2} \equiv 0$, and this is not zero so $G_{\sigma}$ is not a multiple of the identity. (We thank the referee for contributing this crucial case.) Hence for the perturbation $\rho(\tau)=\rho+\tau \sigma$ with the appropriate $\sigma$ as above, at least two of the $\alpha_{j}$ must be distinct, thus concluding the proof of the Lemma and Theorem 2 .

\section{REFERENCES}

1. J. H. Albert, Nodal and critical sets for eigenfunctions of elliptic operators, Proc. Sympos. Pure Math., vol. 23, Amer. Math. Soc., Providence, R. I., 1973, pp. 71-78.

2. - Topology of the nodal and critical point sets for eigenfunctions of elliptic operators, Thesis, M. I. T., Cambridge, Mass., 1971.

3. L. Bers, F. John and M. Schechter, Partial differential equations, Lectures in Appl. Math., vol. 3, Interscience, New York, 1964. MR 29 \#346.

4. R. Courant and D. Hilbert, Methods of mathematical physics. Vols. I, II, Interscience, New York, 1953, 1962. MR 16, 426; 25 \#4216.

5. F. Rellich, Perturbation theory of eigenvalue problems, Gordon and Breach, New York, 1969. MR 39 \#2014.

6. K. Uhlenbeck, Generic properties of eigenfunctions (manuscript).

DEP ARTMENT OF MATHEMATICS, TUFTS UNIVERSITY, MEDFORD, MASSACHUSETTS 02155 